\title{
Dynamic substructuring and reanalysis methods in a surrogate-based design optimization environment
}

\author{
D. Akçay Perdahcioğlu • H. J. M. Geijselaers • \\ M. H. M. Ellenbroek • A. de Boer
}

Received: 28 December 2010 / Revised: 17 May 2011 / Accepted: 10 June 2011 / Published online: 7 July 2011

(C) The Author(s) 2011. This article is published with open access at Springerlink.com

\begin{abstract}
In light weight structure design, vibration control is necessary to meet strict stability requirements and to improve the fatigue life of structural components. Due to ever-increasing demands on products, it is generally more convenient to include vibration prerequisites in a design process instead of using vibration control devices on fixed designs. One of the main difficulties associated to design optimization of complex and/or large structures is the numerous computationally demanding Finite Element (FE) calculations. The objective of this research is to present a novel strategy for efficient and accurate optimization of vibration characteristics of structures. In the proposed strategy, a sub-structuring method is utilized. The FE model of the complete structure is partitioned, reduced and then reassembled. This increases the computational efficiency of dynamic analyses. Moreover, this method is coupled with a novel reanalysis technique to speed up the repeated structural analyses. These methods are finally embedded in a surrogate-based design optimization procedure. An academic test problem is used for the validation of this novel approach.
\end{abstract}

Keywords Dynamic substructuring - Reanalysis methods . Surrogate-based optimization

This research is presented in 27th international congress of the Aeronautical Sciences, ICAS, 19-24 Sept. 2010, Nice, France.

D. Akçay Perdahcıoğlu ( $\varangle)$ · H. J. M. Geijselaers · A. de Boer Faculty of Engineering Technology, Applied Mechanics, University of Twente, P.O. Box 217, 7500 AE Enschede,

The Netherlands

e-mail: d.akcay@ctw.utwente.nl

M. H. M. Ellenbroek

Dutch Space B.V., P.O. Box 32070, 2303 DB Leiden, The Netherlands

\section{Introduction}

During a design process, analysis and, when necessary, modification of the vibration characteristics of a structure are important to improve product quality, to increase process efficiency, to prolong life-time, to increase reliability and safety of the structure. Thanks to the developments in computer technology and in numerical analysis methods, particularly the Finite Element (FE) method, a complex and/or a large structure can be analyzed extensively in the computer environment long before its first prototype is built. To improve its design and to find an optimal configuration, in theory it is possible to directly couple its Finite Element (FE) model with an optimization method. However, in practice this may not be feasible due to the required number of the FE calculations and the corresponding computational costs. Analysis time grows rapidly with the amount of details in the FE model. If the vibration characteristics of a structure need to be improved by modifying the design of the detailed sections, long running analyses are a bottleneck in optimization. Therefore, taking advantage of effective structural analysis methods is as important as utilizing efficient numerical optimization techniques.

For the analysis of structures having a large number of degrees of freedom (d.o.f.), a large system of equations must be solved. In most dynamic analysis problems, the lower natural frequencies and the corresponding modes are more interesting than the higher ones. This is because these modes tend to dominate the dynamic behavior of structures and resonance effects are more severe at the lower natural frequencies. In these problems, the required number of d.o.f. to solve the system accurately is much less than the number of the actual d.o.f. Therefore, condensing the FE models of these structures before the dynamic response analysis is a very frequently used strategy. Reduction is achieved 
by employing a few preselected basis vectors which span the solution space of the approximation. This is known as the reduced basis approach (Kirsch 2002, 2008). The socalled Component Mode Synthesis (CMS) technique is both a reduction and a sub-structuring method which has been utilized since the 1960s for the dynamic analysis of complex and/or large structures. CMS consists of breaking up a large structure into several substructures, obtaining reduced order system matrices of each component and then assembling these matrices to obtain the reduced order system matrices of the entire structure. Depending on the type of the boundary conditions applied on the component interface nodes, CMS can be grouped into fixed interface methods (Craig and Bampton 1968; Hurty 1965), free interface methods (Goldman 1969; Mac Neal 1971; Markovic et al. 2007; Rixen 2002; Rubin 1975) and loaded interface methods (Benfield and Hruda 1971). In this study; the CraigBampton (CB) method, a fixed interface CMS method, is considered to improve the computational efficiency of structural analyses. It is highly regarded for its simplicity and computational stability. The benefits of employing the CB method in an optimization process can be summarized as: (1) Reduction in the total number of d.o.f. leads to fast analysis of the complete structure while, the accuracy of the analyses is preserved within the low-frequency range. (2) Independent condensation of each substructure encourages parallel processing. (3) Only the modified components need to be reanalyzed and coupled with the already computed unmodified ones. (4) For structures having repeated components, modeling one of these components is sufficient.

For reducing the analysis time even further during optimization, employing reanalysis methods can also be very useful. The idea behind these methods is to use the knowledge of the initial model for evaluating the structural response due to the modifications in the design variables. Therefore, solving a complete set of new equations is avoided. More information on reanalysis methods for the eigenvalue problems can be found in (Bouazzouni 1997; Kirsch and Bogomolni 2004; Masson et al. 2006; Cerulli et al. 2007). In this research; a novel approach (Akçay Perdahcioğlu et al. 2010), presenting the integration of two reanalysis methods into the $\mathrm{CB}$ method, is utilized. It is important to clarify that, this study focuses only on the modal and the harmonic response analyses of structures.

Direct coupling of an FE model with numerical optimization algorithms is inevitable for problems which have many design variables, i.e. large scale strongly coupled optimization problems. If it is feasible to calculate the derivatives, gradient-based algorithms (Haftka and Gürdal 1992; Jacobs et al. 2004; Barthelemy and Haftka 1993; Svanberg 1987) are the most suitable because they require less function evaluations (FE analyses) than the derivative-free algorithms. On the other hand, efficient and accurate calculation of the derivatives are remaining issues in their application. The finite difference approximations are the simplest and the easiest methods to calculate derivatives (Kirsch 1994; Van Keulen et al. 2005). Unfortunately, they require numerous repeated FE analyses and high computational costs particularly during the optimization of large structural systems with many design variables. Analytical methods provide exact solutions and reduce the number of the FE analysis calls drastically. However they cannot be easily obtained for many complex problems (Maute et al. 2001). Moreover, access to the source code of the FE software is required for their implementation.

For small-scale optimization problems (i.e. problems with a small number of design variables), where one wants to explore the design domain globally, Surrogate-based Optimization (SBO) can be a good alternative to algorithms based on direct coupling. The motivation of SBO is to replace expensive-to-evaluate FE models with their fast-toevaluate approximations in optimization problems. These approximations are known as meta-models, surrogate models or response surfaces in the literature. When they are defined on the overall design domain, they are also called global approximations. Meta-models are built to predict the trends in the data collected from an FE model. The data consists of a set of values for the selected design variables and the response of the structure for these design values. Therefore, surrogate models can be considered as highly simplified versions of FE models. Once a surrogate model has been built, it is many orders of magnitude faster to evaluate than the FE model. Thus, it can be effectively employed in global optimization schemes. The number of function evaluations in an optimization algorithm is not a big issue due to the simplicity of the surrogate models. Analytical derivatives of the FE models are not required for building the surrogates. Additionally, analytical derivatives of the surrogates are not essential during optimization. Derivatives of these can accurately be calculated by the finite difference approximation. When an optimization algorithm is directly coupled with an FE model, evaluation of the model is done sequentially during the search of an optimum. On the other hand, the FE model is only required for generating data for meta-modeling in SBO. Hence, the data can be gathered all at once by parallel processing. The SBO methods differ from each other by the followed approaches during the generation of the surrogate model and the utilized algorithms at the optimization step (Queipo et al. 2005). For instance, Jones et al. (1998) present a method based on the Kriging approximation at the surrogate modeling phase and the Branch and Bound (a mixed integer nonlinear programming) algorithm for optimization. Berke and Hajela (1992) use the NN approximations for surrogate modeling and the gradient-based algorithms for optimization. Another SBO method is the one proposed by Booker 
et al. (1998) in which the Kriging approximations are utilized for meta-modeling and the pattern search (a derivative free optimization) method is employed during the search of an optimum.

Data generation is the challenging step of surrogate modeling. For obtaining certain accuracy, the total number of the data should be sufficient. On the other hand, with an increasing number of the design variables, the required number of data grows rapidly. Accordingly, the number of the $\mathrm{FE}$ analysis calls increases significantly. In order to reduce this computational burden, an SBO method is proposed by Akçay Perdahcioğlu et al. (2009) for optimizing the dynamic behavior of structures where global approximations are utilized as surrogates. In the method, CraigBampton (CB) method is used for offering solutions to one of the major difficulties in $\mathrm{SBO}$, the analysis time. Neural Networks (NNs) are the meta-modeling technique used in the SBO method. The search for the global optimum is done by the Multi-Level Hybrid Optimization (MLHO) scheme. Genetic Algorithms and Sequential Quadratic Programming are the numerical optimization methods employed in MLHO. In this research, the SBO method proposed by Akçay Perdahcioğlu et al. (2009) is coupled with a novel reanalysis approach (Akçay Perdahcioğlu et al. 2010) with which it is aimed to increase the computational efficiency of the SBO strategy further.

The structure of the paper is organized as follows: The CB method and the reanalysis approach are introduced briefly in Sections 2 and 3. The proposed SBO method is introduced in Section 4. The final section includes the demonstration of the introduced concepts.

\section{Craig-Bampton method}

Assume that an FE model of a structure is constructed on a domain $\Omega$ and is divided into $S$ non-overlapping substructures such that each component is defined on the sub-domain $\Omega^{c}$. Thus, excepting the nodes on the interface boundaries, each node belongs to one and only one component. The linear dynamic behavior of an undamped component, labeled $c$, is governed by the equations,

$$
\begin{aligned}
& {\left[\begin{array}{ll}
\mathbf{M}_{\mathrm{ii}}^{c} & \mathbf{M}_{\mathrm{ib}}^{c} \\
\mathbf{M}_{\mathrm{bi}}^{c} & \mathbf{M}_{\mathrm{bb}}^{c}
\end{array}\right]\left\{\begin{array}{l}
\ddot{\mathbf{d}}_{\mathrm{i}}^{c} \\
\ddot{\mathbf{d}}_{\mathrm{b}}^{c}
\end{array}\right\}+\left[\begin{array}{cc}
\mathbf{K}_{\mathrm{ii}}^{c} & \mathbf{K}_{\mathrm{ib}}^{c} \\
\mathbf{K}_{\mathrm{bi}}^{c} & \mathbf{K}_{\mathrm{bb}}^{c}
\end{array}\right]\left\{\begin{array}{l}
\mathbf{d}_{\mathrm{i}}^{c} \\
\mathbf{d}_{\mathrm{b}}^{c}
\end{array}\right\}} \\
& =\left\{\begin{array}{l}
\mathbf{f}_{\mathrm{i}}^{c} \\
\mathbf{f}_{\mathrm{b}}^{c}
\end{array}\right\}+\left\{\begin{array}{c}
\mathbf{0} \\
\mathbf{g}_{\mathrm{b}}^{c}
\end{array}\right\}
\end{aligned}
$$

where " $\mathrm{i}$ " and " $\mathrm{b}$ " refer to interior and boundary, respectively. In the formulation, $\mathbf{M}^{c}, \mathbf{K}^{c}$ and $\mathbf{d}^{c}$ are respectively the mass matrix, the stiffness matrix and the vector of the local d.o.f. of the component. The vector $\mathbf{f}^{c}$ represents the external loads, and the vector $\mathbf{g}^{c}$ represents the interface loads between the component $c$ and the neighboring components that ensure compatibility at the interfaces.

For reducing the size of the component matrices, $\mathbf{K}^{c}$ and $\mathbf{M}^{c}$, a subspace spanned by the columns of $\mathbf{T}^{c}$ is built in such a way that the solution of (1) can be written in the form:

$\mathbf{d}^{c} \approx \mathbf{T}^{c} \mathbf{q}^{c}$

where $\mathbf{q}^{c}$ is a vector of generalized coordinates and $\operatorname{dim}\left(\mathbf{q}^{c}\right) \ll \operatorname{dim}\left(\mathbf{d}^{c}\right)$. $\mathbf{T}^{c}$ is referred to as a reduction basis, a transformation matrix or a Ritz basis.

The $\mathrm{CB}$ reduction basis is obtained utilizing the fixed interface normal modes, $\left[\begin{array}{ll}\boldsymbol{\Phi}_{\mathrm{i}}^{c} & \mathbf{0}\end{array}\right]^{\mathrm{T}}$, and the constraint modes, $\left[\begin{array}{ll}\boldsymbol{\Psi}_{\mathrm{ib}}^{c} & \mathbf{I}_{\mathrm{bb}}\end{array}\right]^{\mathrm{T}}$.

The fixed interface normal modes describe the internal dynamic behavior of a substructure. These modes are calculated by restraining all d.o.f. at the interface and solving an undamped free vibration problem

$$
\left(\mathbf{K}_{\mathrm{ii}}^{c}-\omega_{j}^{2} \mathbf{M}_{\mathrm{ii}}^{c}\right)\left\{\Phi_{\mathrm{i}}^{c}\right\}_{j}=0 \quad j=1,2, \ldots, N_{\mathrm{T}}
$$

where $\omega_{j},\left\{\Phi_{\mathrm{i}}^{c}\right\}_{j}$ are the $j$ th natural frequency and the corresponding mode shape respectively, and, $N_{\mathrm{T}}$ is the truncated number of the normal modes which is usually a lot less than the actual number.

The motion on the substructure interfaces, the propagation of the forces between substructures and the necessary information about the rigid body motions are defined by the constraint modes. These modes are calculated by statically imposing a unit displacement to the interface d.o.f. one by one while keeping the displacement of the other interface d.o.f. zero and assuming that there are no internal reaction forces, i.e.,

$\left[\begin{array}{ll}\mathbf{K}_{\mathrm{ii}}^{c} & \mathbf{K}_{\mathrm{ib}}^{c} \\ \mathbf{K}_{\mathrm{bi}}^{c} & \mathbf{K}_{\mathrm{bb}}^{c}\end{array}\right]\left[\begin{array}{l}\boldsymbol{\Psi}_{\mathrm{ib}}^{c} \\ \mathbf{I}_{\mathrm{bb}}^{c}\end{array}\right]=\left[\begin{array}{c}\mathbf{0}_{\mathrm{ib}}^{c} \\ \mathbf{R}_{\mathrm{bb}}^{c}\end{array}\right]$.

In (4), $\mathbf{R}_{\mathrm{bb}}^{c}$ is a matrix with the unknown reaction forces acting on the interface.

The Craig-Bampton transformation matrix $\mathbf{T}_{\mathrm{CB}}^{c}$ for component $c$ is defined as,

$\mathbf{T}_{\mathrm{CB}}^{c}=\left[\begin{array}{cc}\boldsymbol{\Phi}_{\mathrm{i}}^{c} & \boldsymbol{\Psi}_{\mathrm{ib}}^{c} \\ \mathbf{0} & \mathbf{I}_{\mathrm{bb}}\end{array}\right]$.

After defining the $\mathrm{CB}$ reduction basis $\mathbf{T}_{\mathrm{CB}}^{c}$, first, the right-hand side of (2) is substituted into (1) and then, (1) is pre-multiplied by $\mathbf{T}_{\mathrm{CB}}^{c}{ }^{\mathrm{T}}$. Hence, the reduced matrices of each component are defined by: $\overline{\mathbf{K}}^{c}=\mathbf{T}_{\mathrm{CB}}^{c}{ }^{\mathrm{T}} \mathbf{K}^{c} \mathbf{T}_{\mathrm{CB}}^{c}$, $\overline{\mathbf{M}}^{c}=\mathbf{T}_{\mathrm{CB}}^{c}{ }^{\mathrm{T}} \mathbf{M}^{c} \mathbf{T}_{\mathrm{CB}}^{c}$. The external loads and the interface loads are $\overline{\mathbf{f}}^{c}=\mathbf{T}_{\mathrm{CB}}^{c}{ }^{\mathrm{T}} \mathbf{f}^{c}$ and $\overline{\mathbf{g}}^{c}=\mathbf{T}_{\mathrm{CB}}^{c}{ }^{\mathrm{T}} \mathbf{g}^{c}$, respectively.

In the CB method, the assembly of the components is done using the compatibility of the interface d.o.f. This implies matching FE meshes at the interfaces. 


\section{Reanalysis methods for updating the $\mathrm{CB}$ reduction basis}

When a substructure is reanalyzed with modified design parameters in an optimization algorithm, the static and the dynamic properties of the component are not the same as the initial ones anymore. Consequently, the reduction basis is also different. One can either reuse the reduction basis of the initial component or compute a new basis for the condensation of the matrices of the modified component. The first option usually leads to inaccurate results. The second one requires solving free vibration problems and performing static analyses which are computationally demanding for complex structures. Alternative and fast methods that can be used for updating the $\mathrm{CB}$ reduction basis during optimization are introduced in this section. These methods are suitable for sizing optimization problems.

\subsection{Updating the fixed interface normal modes}

For updating the initial fixed interface normal mode set of a substructure, the Enriched CB (ECB) method proposed by Masson et al. (2006) is utilized. In this method, first, the residual forces $\mathbf{R}_{\mathrm{L}}=\left[\mathbf{f}_{\boldsymbol{\Delta}}\left(\omega_{1}\right), \ldots, \mathbf{f}_{\boldsymbol{\Delta}}\left(\omega_{N_{\mathrm{T}}}\right)\right]$ are calculated. These act on the initial substructure due to the design modifications where

$\mathbf{f}_{\boldsymbol{\Delta}}\left(\omega_{j}\right)=-\left[\boldsymbol{\Delta} \mathbf{K}_{\mathrm{ii}}-\omega_{j}^{2} \boldsymbol{\Delta} \mathbf{M}_{\mathrm{ii}}\right]\left\{\Phi_{\mathrm{i}}\right\}_{j}$,

$\Delta \mathbf{K}_{\mathrm{ii}}, \boldsymbol{\Delta} \mathbf{M}_{\mathrm{ii}}$ stand for the introduced modifications on $\mathbf{K}_{\mathrm{ii}}$ and $\mathbf{M}_{\mathrm{ii}}$ and, $\omega_{j},\left\{\Phi_{\mathrm{i}}\right\}_{j}$ are the $j$ th natural frequency and the corresponding mode shape of the modified substructure, respectively. Afterwards, these residual forces are used to define a correction to the initial displacement field. However, their exact calculation is not possible with only knowledge of the initial substructure data. Therefore, they are approximated by, first, computing the residual forces

$\hat{\mathbf{R}}_{\mathrm{L}}=\left[\hat{\mathbf{f}}_{\boldsymbol{\Delta}}\left(\omega_{1}\right), \ldots, \hat{\mathbf{f}}_{\boldsymbol{\Delta}}\left(\omega_{N_{\mathrm{T}}}\right)\right]$

acting on the modified structure where

$\hat{\mathbf{f}}_{\boldsymbol{\Delta}}\left(\omega_{j}\right)=-\left[\boldsymbol{\Delta} \mathbf{K}_{\mathrm{ii}}-\left\{\omega_{j}^{0}\right\}^{2} \boldsymbol{\Delta} \mathbf{M}_{\mathrm{ii}}\right]\left\{\Phi_{\mathrm{i}}^{0}\right\}_{j}$

The fixed interface normal modes and the corresponding eigenvalues of the initial model are represented by $\left\{\Phi_{i}^{0}\right\}_{j}$ and $\left\{\omega_{j}^{0}\right\}^{2}$, respectively. Then, the approximate residual forces are defined as

$\mathbf{f}_{\boldsymbol{\Delta}}\left(\omega_{j}\right) \approx y_{1} \hat{\mathbf{f}}_{\boldsymbol{\Delta}}\left(\omega_{1}\right)+\ldots+y_{N_{T}} \hat{\mathbf{f}}_{\boldsymbol{\Delta}}\left(\omega_{N_{T}}\right)$.
The matrix $\hat{\mathbf{R}}_{\mathrm{L}}$ that involves the approximate residual forces can be utilized to replace $\mathbf{R}_{\mathrm{L}}$ and the corrections to the displacement field can be imposed using them. The essential idea of doing this is: if the subspace spanned by $\hat{\mathbf{R}}_{\mathrm{L}}$ does not contain the exact residual force vectors with respect to a specific design modification, it may at least contain a reasonable representation of these vectors. The approximate correction matrix $\tilde{\mathbf{R}}_{\mathrm{D}}$ is then defined as

$\tilde{\mathbf{R}}_{\mathrm{D}}=\mathbf{K}_{\mathrm{ii}}^{-1} \tilde{\mathbf{R}}_{\mathrm{L}}$

where $\tilde{\mathbf{R}}_{\mathrm{L}}$ is the reconditioned form of $\hat{\mathbf{R}}_{\mathrm{L}}$ by Singular Value Decomposition. Finally, the initial fixed interface normal mode set is enriched by $\tilde{\mathbf{R}}_{\mathrm{D}}$, that is,

$\boldsymbol{\Phi}=\left[\begin{array}{cc}\boldsymbol{\Phi}_{\mathrm{i}}^{0} & \tilde{\mathbf{R}}_{\mathrm{D}} \\ \mathbf{0} & \mathbf{0}\end{array}\right]$

This extended set of vectors is then used in the CB transformation matrix for the condensation of the modified component.

\subsection{Updating the constraint modes}

For updating the initial constraint mode set of a substructure, a method based on the Combined Approximations (CA) approach is utilized (Akçay Perdahcıoğlu et al. 2010). In this method, the residual constraint mode matrix, $\Delta \boldsymbol{\Psi}_{\mathrm{ib}}$, is approximated using the conditioned binomial series expansion. A brief description of the procedure is as follows:

The $t$ th residual constraint mode $\left\{\boldsymbol{\Delta} \boldsymbol{\Psi}_{\mathrm{ib}}\right\}_{t}$ is approximated in the space spanned by the vectors of the basis

$\mathbf{H}_{t}=\left[\left\{\boldsymbol{\Delta} \mathbf{r}_{1}\right\}_{t}, \ldots,\left\{\boldsymbol{\Delta} \mathbf{r}_{N_{b}}\right\}_{t}\right], \quad t=1,2, \ldots, N_{\mathrm{s}}$

where

$\left\{\boldsymbol{\Delta} \mathbf{r}_{1}\right\}_{t}=\mathbf{K}_{\mathrm{ii}}^{-1} \mathbf{R}_{t},\left\{\boldsymbol{\Delta} \mathbf{r}_{k}\right\}_{t}=-\mathbf{K}_{\mathrm{ii}}^{-1} \boldsymbol{\Delta} \mathbf{K}_{\mathrm{ii}}\left\{\boldsymbol{\Delta} \mathbf{r}_{k-1}\right\}_{t}$

In the formulation, $k=2,3, \ldots, N_{\mathrm{b}}$ indicates the number of the basis vector (binomial series term), $N_{\mathrm{b}}$ is the total number of the binomial series terms used in the approximation and $\mathbf{R}_{t}$ is the $t$ th column of

$\mathbf{R}=-\Delta \mathbf{K}_{\mathrm{ii}} \boldsymbol{\Psi}_{\mathrm{ib}}^{0}-\Delta \mathbf{K}_{\mathrm{ib}}$

The initial constraint mode matrix is represented by $\boldsymbol{\Psi}_{\mathrm{ib}}^{0}$.

Having defined the basis $\mathbf{H}_{t},\left\{\boldsymbol{\Delta} \boldsymbol{\Psi}_{\mathrm{ib}}\right\}_{t}$ can be approximated as

$$
\begin{aligned}
\left\{\boldsymbol{\Delta} \boldsymbol{\Psi}_{\mathrm{ib}}\right\}_{t} & \approx\left\{\boldsymbol{\Delta} \mathbf{r}_{1}\right\}_{t} y_{t, 1}+\ldots+\left\{\boldsymbol{\Delta} \mathbf{r}_{N_{\mathrm{b}}}\right\}_{t} y_{t, N_{\mathrm{b}}} \\
& =\mathbf{H}_{t} \mathbf{y}_{t}
\end{aligned}
$$


where $\mathbf{y}_{t}^{\mathrm{T}}=\left\{y_{t, 1}, y_{t, 2}, \ldots, y_{t, N_{\mathrm{b}}}\right\}$ is a vector of unknown coefficients. These coefficients can be obtained by solving a linear system of equations

$$
\left[\mathbf{H}_{t}^{\mathrm{T}}\left(\mathbf{K}_{\mathrm{ii}}+\Delta \mathbf{K}_{\mathrm{ii}}\right) \mathbf{H}_{t}\right] \mathbf{y}_{t}=\mathbf{H}_{t}^{\mathrm{T}}\left(-\boldsymbol{\Delta} \mathbf{K}_{\mathrm{ii}}\left\{\boldsymbol{\Psi}_{\mathrm{ib}}^{0}\right\}_{t}-\left\{\boldsymbol{\Delta} \mathbf{K}_{\mathrm{ib}}\right\}_{t}\right)
$$

whose size is much smaller than that of the original one (the original system has the same size as (4)). When this system is solved for $\mathbf{y}_{t}$ and the solution is inserted back into (6), the $t$ th residual constraint mode $\left\{\boldsymbol{\Delta} \boldsymbol{\Psi}_{\mathrm{ib}}\right\}_{t}$ is computed approximately. Performing the above defined operations for each residual constraint mode, the CA approach of the residual constraint mode matrix $\Delta \boldsymbol{\Psi}_{\mathrm{ib}}$ is defined as

$$
\Delta \Psi_{\mathrm{ib}}=\left[\left\{\Delta \boldsymbol{\Psi}_{\mathrm{ib}}\right\}_{1},\left\{\Delta \boldsymbol{\Psi}_{\mathrm{ib}}\right\}_{2}, \ldots,\left\{\Delta \boldsymbol{\Psi}_{\mathrm{ib}}\right\}_{N_{s}}\right]
$$

Hence, the approximate constraint mode matrix is given by

$$
\Psi \approx\left[\begin{array}{c}
\Psi_{\mathrm{ib}}^{0}+\Delta \Psi_{\mathrm{ib}} \\
\mathbf{I}_{\mathrm{bb}}
\end{array}\right]
$$

It is possible to automate the calculation of the constraint modes. To do that,first, a value is assigned to the initial number of the basis vectors in the CA approach. Next, the number of FLoating-point OPerations (FLOPs) is estimated. This number is compared with the number of FLOPs of the exact analysis. The CA approach is used only when it requires less FLOPs than the exact analysis. If it is computationally efficient to be employed, the residual constraint mode matrix $\Delta \boldsymbol{\Psi}_{\mathrm{ib}}$ is calculated using CA. The accuracy of the approximation is verified. If the accuracy is not satisfactory and the number of FLOPs of CA is still less than the exact analysis when a new vector is added to the basis $\mathbf{H}_{t}$, it is extended with this vector. The reanalysis is performed again. Otherwise, the constraint modes are computed with the exact analysis (Akçay Perdahcioğlu et al. 2010).

\section{Surrogate-based optimization method}

The solution process of the SBO method is as illustrated in Fig. 1. It starts with the problem analysis which involves, first, understanding the problem under consideration. Then, selection of the design variables and parameterization of the computational model are carried out. Finally the objective function and the constraints are defined.

The second step is to generate the surrogate model. Here, first, a set of sample points is selected from the design space which is called Design of Experiments (DOE). In the method, Latin Hypercube Sampling (LHS) scheme is utilized to generate the DOE set. Afterwards, for each sample point, the FE model is run and data is gathered for training the surrogate. At the analysis step, the Craig-Bampton (CB) method is used as a CMS technique. Furthermore, reanalysis methods are considered for efficient calculation of the CB transformation matrices of the modified components. The followed steps at the analysis phase are shown schematically in Fig. 2. For the dynamic analysis of a structure, first, the complete structure is divided into components. Then the parameterized FE model of each component is built. If there are similar components, only one of them is modeled. Afterwards, the design values of the complete structure are distributed to components based on the design variables captured in the component models. An FE model standing for similar components may get multiple configurations for its design variables. The next step is the calculation of the reduced system matrices of each component for the assigned design values. In the proposed scheme, libraries are used to store the information about the already analyzed components. Hence, unnecessary analyses are prevented. Before generating the system matrices of a given component design, first, the corresponding library is checked. If the requested information is not there, it is computed and stored in the library. In the computation, first of all, the transformation matrix, consisting of the normal and the constraint modes, is calculated. The normal modes

Fig. 1 Schematic illustration of the SBO method

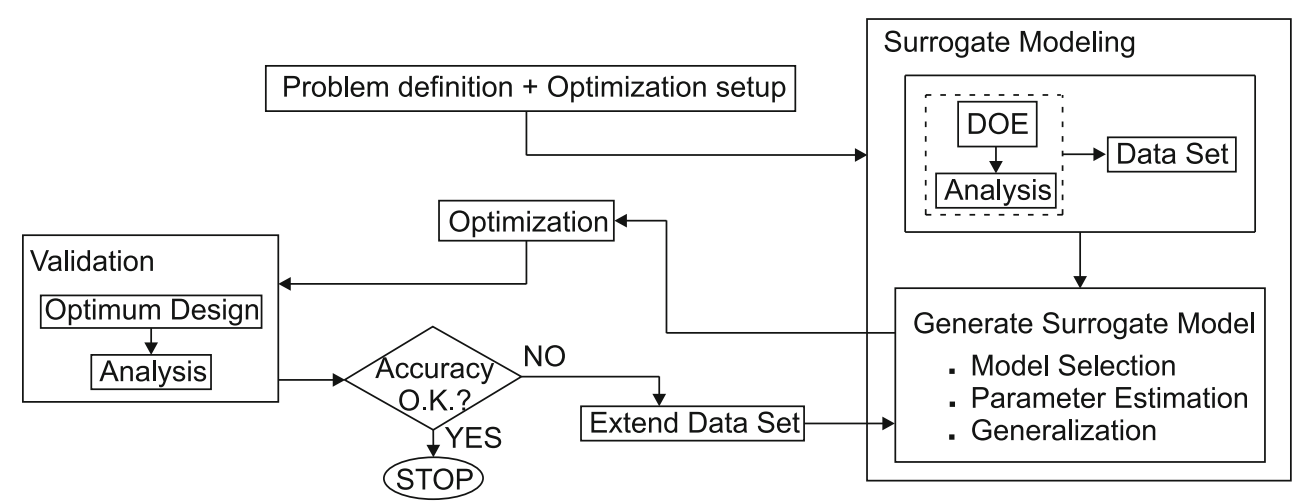


Decompose structure into components (One model for similar components)

Gather system matrices from component libraries for a given structure design and Assemble

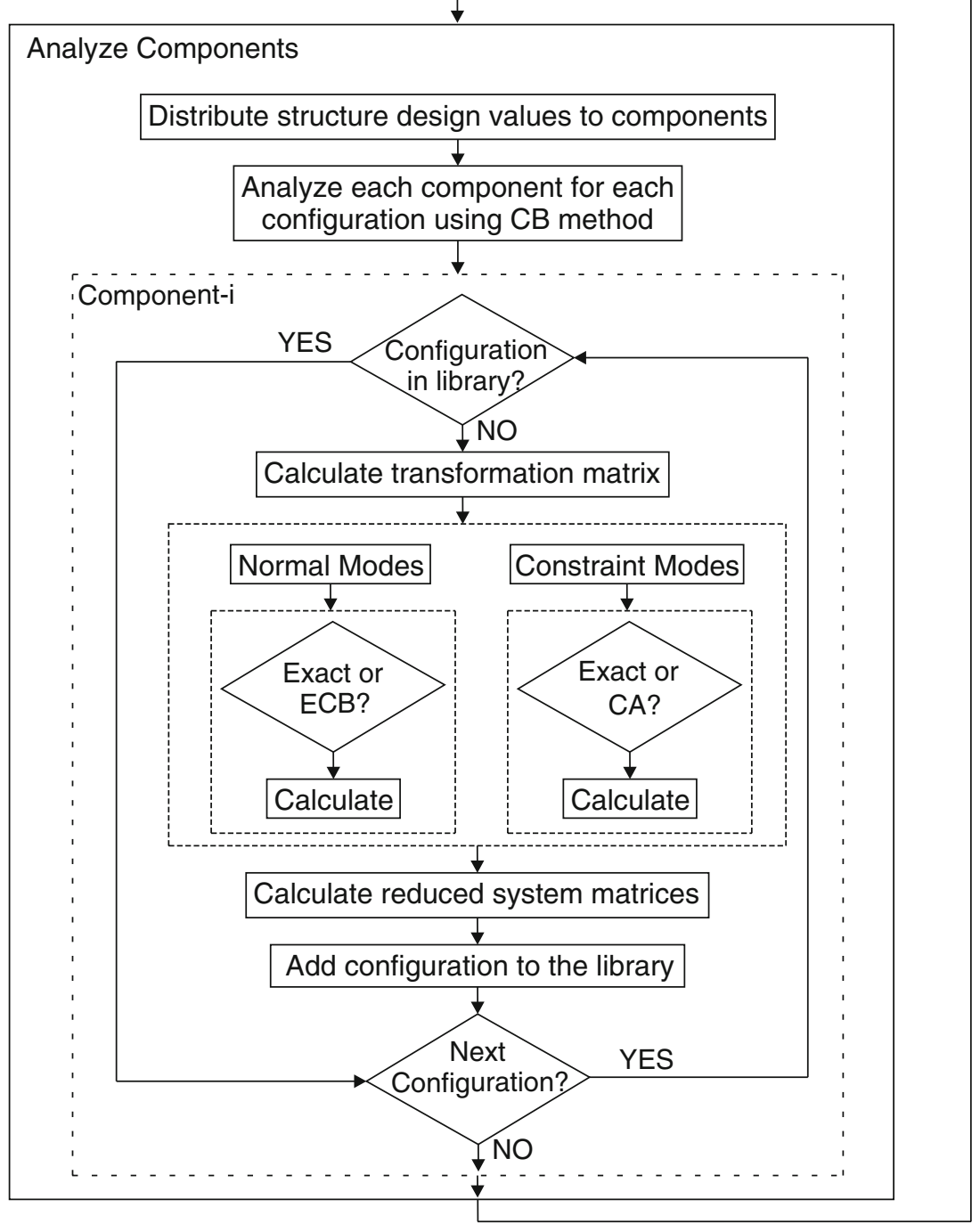

Fig. 2 Schematic illustration of the analysis step of the SBO method

can be computed either using the exact analysis methods or using the Enriched Craig-Bampton (ECB) method. Unfortunately, there is no automated switch from ECB to the exact methods based on the accuracy and/or the computational efficiency of ECB. On the other hand, calculation of the constraint modes, either by the exact or the approximate methods, can be automated. The approximate constraint modes are calculated using the Combined Approximations (CA) approach. After the transformation matrix is determined, the reduced component matrices are computed. The given component design, its transformation and the reduced matrices are saved in the component library. This procedure is repeated for each component and the corresponding configurations. This ensures that all the necessary information to generate the reduced system matrices of the complete structure is readily available in the libraries for further use. Thereafter, the stored reduced matrices are gathered from the libraries for the given structure design and assembled to obtain the reduced matrices of the entire structure. Finally, the dynamic analysis of the structure is performed.

After defining the data set, a suitable meta-modeling approach is selected and the unknown parameters of the chosen meta-model are determined using the available data. In the proposed method, Back-propagation Neural Networks are employed for this purpose. The possible overfitting problems are tried to be prevented utilizing the 
Bayesian regularization of Mackay (1992). Details about the surrogate modeling approach can be found in (Akçay Perdahcioğlu et al. 2009).

Having generated the surrogate model, the next step is the optimization where the global optimum is sought using a Multi-Level Hybrid Optimization (MLHO) scheme. In MLHO, a stochastic derivative-free global optimization method, the Genetic Algorithm (GA), is employed to locate the global optimum. Afterwards, a gradient-based method, Sequential Quadratic Programming, is initialized with the solution of GA to find an exact optimum solution.

Since the calculated optimum is not directly related with the FE model but the surrogate model, the results need to be validated. In order to do that, the response of the FE model is obtained at the computed optimum design values. This is then compared with the response of the surrogate model for the same design values. If the accuracy is acceptable, the scheme is stopped. Otherwise, the data set is extended with the optimum design values and the corresponding response of the FE model. New parameters for the selected surrogate model are computed using the extended data set and the optimization step is repeated. This procedure is iterated until the validation results are acceptable.

\section{Demonstration of the concepts}

For the demonstration of the introduced concepts, an idealized fuselage structure, shown in Fig. 3, is utilized. The structure is composed of eight identical components and it is free at the boundaries. A component consists of a cylinder skin including a floor panel, frames and stiffeners whose geometry is as illustrated in Fig. 3.

The reduced system matrices of the entire structure are obtained by only modeling one component. The FE model of a component is generated in the commercial FE software ANSYS. Its system matrices are calculated for the defined design variables and then they are transferred to MATLAB.
For obtaining the reduced system matrices of the components, first of all the transformation matrices are computed and afterwards condensation is performed. In the transformation matrices 18 fixed interface normal modes are used. The number of the nodes on one interface of a component is 37 (each having 6 d.o.f.). After the reduced matrices of all the components are obtained, these matrices are assembled and the reduced system matrices of the entire structure are gathered.

The skin, floor and frames are modeled using a 4-node shell element which has 6 d.o.f. at each node and is suitable to analyze thin to moderately thick shell structures. The stiffeners with I cross-section are modeled with a three dimensional beam element which has 6 d.o.f. at each node. It allows different cross sections and permits the end nodes to be offset from the centroidal axes of the beam. The cross section width and height of the stiffeners $\left(h_{s}\right)$ in the components (see Fig. 3) are defined as the design variables and all the stiffeners of a component are assumed to have the same design values. Therefore, there exist 8 design variables in total in the overall structure. Each component has one design variable. For the initial design $\mathrm{h}_{\mathrm{s}_{i}}, i=1,2, \ldots, 8$ are set to $0.05 \mathrm{~m}$.

There is a harmonic force acting on the structure. The applied load has an amplitude of $100 \mathrm{kN}$ and is in the $y$ direction. It is applied on the top interface node of the 4th component $\mathrm{C}_{4}$ and the 5 th component $\mathrm{C}_{5}$ as shown in Fig. 3 . For the harmonic response analysis, structural damping with an energy dissipation of $3 \%$ is assumed which is imposed directly on the reduced stiffness matrix of the structure.

For the harmonic response analysis, focus is on the frequency range of $10-30 \mathrm{~Hz}$. This interval involves the first bending frequency of the initial design. Figure 3 shows the mode shape of this frequency. The objective is to reduce the amplitude of the displacement response in this frequency range, thereby decreasing the displacement response of the structure for the first bending mode. The nodes that lie on the top and the bottom interface of the components are

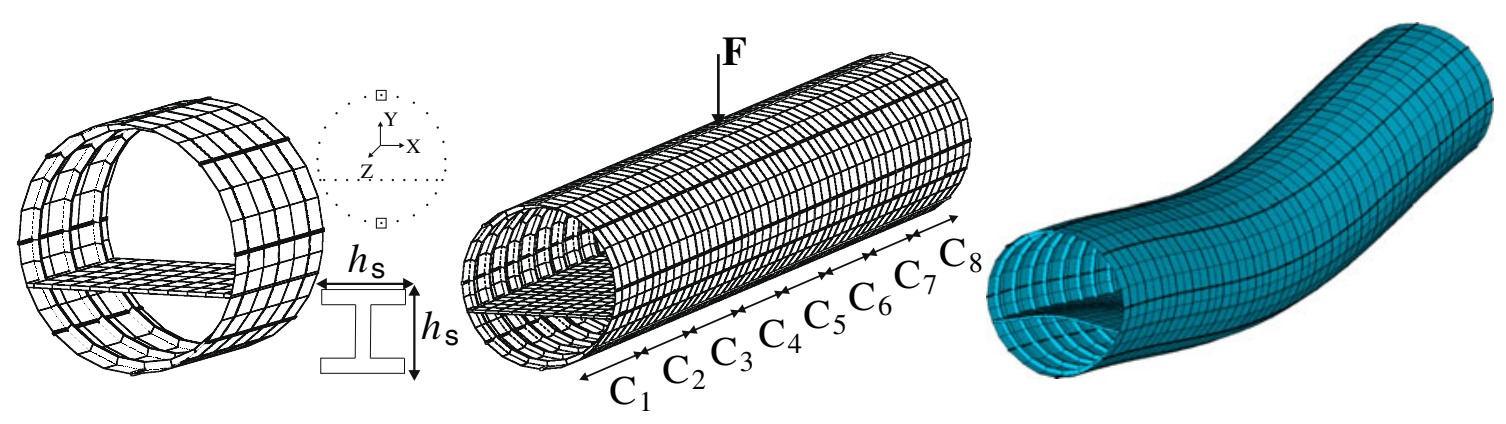

Fig. 3 Test problem. (Left) Component model, nodes on a substructure interface, cross-section of a stiffener, (Middle) Selected structure under applied force, (Right) First bending mode of the initial design 

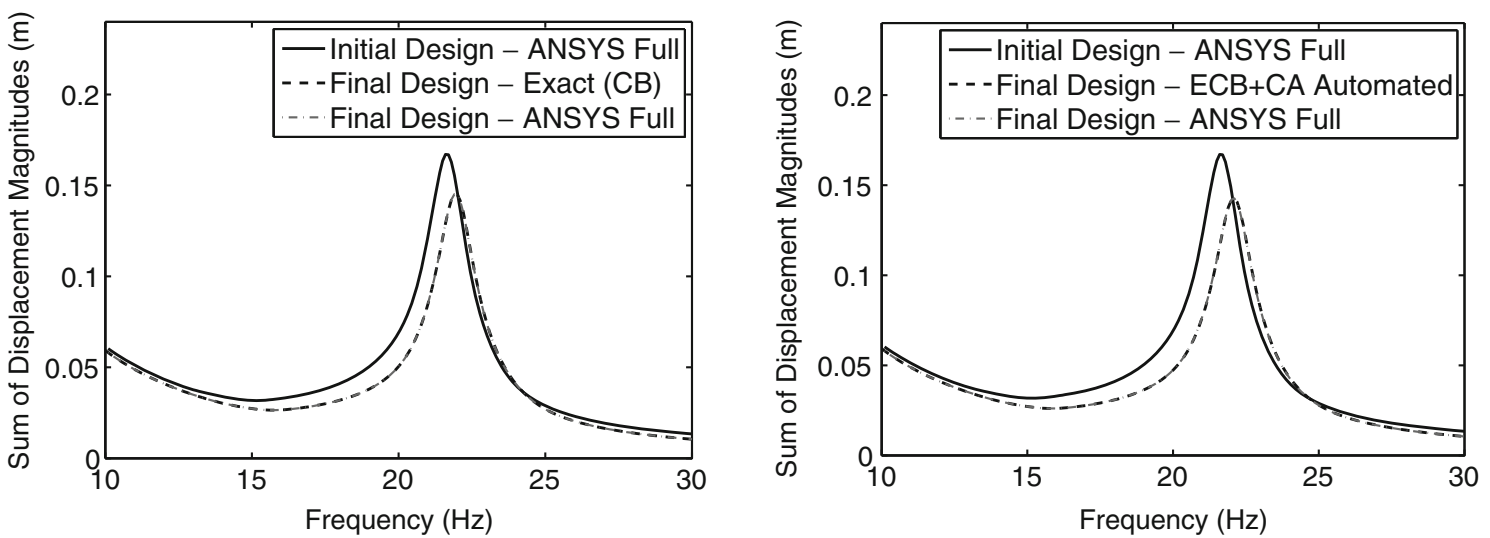

Fig. 4 Results of the test problem. Left The CB transformation matrices are computed by the Exact approach in the SBO method, Right The CB transformation matrices are computed by the ECB+CA Automated approach in the SBO method

selected to prescribe the objective function. The left illustration in Fig. 3 shows the nodes corresponding to the interface of a component. The selected nodes are identified with squares around them. The displacement magnitudes in the $y$-direction are computed for these nodes in the frequency range of $10-30 \mathrm{~Hz}$ and then summed up. The response curve that represents the "frequency-displacement magnitude" relationship of the initial design is plotted in Fig. 4. The results displayed in the figure are obtained by the full FE analysis performed in ANSYS.

The objective function of the problem is defined as minimizing the total area, $\mathrm{A}(\mathbf{h})$, beneath the response curve. This area is $0.95 \mathrm{~m} . \mathrm{Hz}$ for the initial design.

The constraints of the problem are as follows:

- Keep the first bending frequency, $f_{7}$, around $22 \mathrm{~Hz}$. This constraint is defined as $21.98 \leq f_{7}(\mathbf{h}) \leq 22.02$.

- Keep the total final mass of the stiffeners less than the total initial mass of the stiffeners. This is given as, $\sum_{i=1}^{8}\left[\rho \mathrm{V}_{i}\left(\mathrm{~h}_{\mathrm{s}_{i}}\right)\right] \leq 23$ where $\mathrm{V}_{i}\left(\mathrm{~h}_{\mathrm{s}_{i}}\right)$ is the total volume of the stiffeners in component $\mathrm{C}_{i}$ and $\rho$ is the density of the stiffeners.

- Preserve the mode shape of the first bending frequency. This is assured by the MAC criterion. $\operatorname{MAC}_{7}(\mathbf{h}) \geq 0.9$.

- Constraints on the design variables: $\mathrm{h}_{\mathrm{s}_{j}}-\mathrm{h}_{\mathrm{s}_{(9-j)}} \leq 10^{-4}$, $\mathrm{h}_{\mathrm{s}_{(9-j)}}-\mathrm{h}_{\mathrm{s}_{j}} \leq 10^{-4}, \quad j=1,2, \ldots, 4 .^{1}$

- The upper and the lower bounds for the design variables are selected as $0.01 \leq \mathrm{h}_{\mathrm{s}_{i}} \leq 0.1, \quad i=1,2, \ldots, 8$.

In the optimization problem, three surrogate models are used. These surrogates stand for $\mathrm{A}(\mathbf{h}), f_{7}(\mathbf{h})$ and $\mathrm{MAC}_{7}(\mathbf{h})$.

The DOE set $\mathbf{D}_{\mathrm{T}}$ of the whole structure has 81 designs where each design defines a new structure configuration.

\footnotetext{
${ }^{1}$ This problem has many local solutions which lead to similar optimum values. By these constraints, design space is restricted.
}

At the analysis step of the SBO method, the transformation matrix of each modified component is computed using one of the following methods:

Exact The fixed interface normal modes and the constraint modes of the Craig-Bampton (CB) transformation matrix are computed by exact analysis methods all over again.

ECB+CA Automated The initial fixed interface normal mode set is extended using the Enriched Craig-Bampton (ECB) method. For the calculation of the constraint modes, the automated update scheme defined in Section 3 is used. The minimum number of the basis vectors are set to 3 in the CA approach.

After the transformation matrix of a component is calculated using one of the above methods, condensation of the component matrices are performed. The size of the coupled system matrices for Full FE, CB and ECB+CA Automated models are $14334 \times 14334,1698 \times 1698$ and $1842 \times 1842$, respectively.

The responses, $\mathrm{A}(\mathbf{h}), f_{7}(\mathbf{h})$ and $\mathrm{MAC}_{7}(\mathbf{h})$, of the structure for each configuration in $\mathbf{D}_{\mathrm{T}}$ are calculated using the assembled reduced component system matrices and the training data sets are gathered for meta-modeling.

Three separate libraries are used for storing the transformation, the reduced stiffness and the reduced mass matrices of each new component design. The first library is for component $\mathrm{C}_{1}$, the second one is for components $\mathrm{C}_{2}, \mathrm{C}_{3}, \ldots, \mathrm{C}_{7}$ and the third one is for $\mathrm{C}_{8}$.

The optimization problem is solved twice. First, the Exact approach is used for the calculation of the transformation matrices during the analysis step of the SBO method. In the second solution, instead of the Exact approach, the ECB+CA Automated approach is used. The performance of the SBO method is evaluated regarding the accuracy of the results and the computation time. 
Table 1 Summary of the results of the test problem

\begin{tabular}{llll}
\hline Initial & & Final-Exact & Final-ECB+CA Automated \\
\hline$[0.05,0.05,0.05,0.05$, & Design (m) & {$[0.01,0.01,0.058,0.1$,} & {$[0.01,0.01,0.063,0.1$,} \\
$0.05,0.05,0.05,0.05]$ & & $0.1,0.058,0.01,0.01]$ & $0.1,0.063,0.01,0.01]$ \\
\hline 0.95 & Area (m.Hz) & 0.8238 & 0.8161 \\
24.0 & Mass (kg) & 21.7 & 22.5 \\
- & Total \# of iterations & 4 & 4 \\
21.67 & $f_{7}(\mathrm{~Hz})$ & 22.02 & 22.02 \\
- & MAC 7 & 0.9834 & 0.9913 \\
- & Computation time & 1 & 0.6993 \\
& (normalized) & & \\
\hline
\end{tabular}

In the test case, $\mathrm{NN}$ model is employed with 25 hidden layer neurons for each surrogate.

The search for optimum is repeated until the relative errors between the responses of the FE model and that of the surrogates are smaller than 0.005 for the computed optimum design values. The relative error is computed with respect to the FE analysis results.

Before calculating the reduced system matrices of the components for the optimum design values, first the libraries are checked for similar component designs. These designs are sought with a relative error tolerance of $10^{-3}$. The relative error is calculated with respect to the investigated optimum design.

\subsection{Results and discussions}

The results are summarized in Table 1. The "frequencydisplacement magnitude" curves that correspond to the final configurations are shown in Fig. 4. To validate the results, the response of the structure is calculated in ANSYS using the full FE analysis for the final design values. These solutions are also presented in Fig. 4.

Both of the final configurations are feasible. These configurations have almost the same design values. The optimal configuration is stiffest in the middle while the stiffness decreases towards the free ends of the structure. The total area beneath the "frequency-displacement magnitude" curve is reduced by almost $14 \%$ in both Exact and ECB+CA Automated.

The total required time for the optimization process decreases around 30\% when the ECB+CA Automated approach is utilized in the SBO method. The computational efficiency of the CA approach depends on the properties of the FE models of the components (Akçay Perdahcioğlu et al. 2010). More specifically, the ratio between the size of the internal stiffness matrix and the number of the constraint modes is the driving factor. While the maximum number of the binomial series terms that can be used for Components $2, \ldots, 7$ is limited to $\approx 2$ terms, this number is $\approx 3$ for Components 1 and 8. This means that, only the Exact analysis are utilized for Components $2, \ldots, 7$ whereas for Components 1 and 8 the $\mathbf{C A}$ approach and the Exact analysis are actively utilized in the CA Automated approach. The selection is made automatically based on both the accuracy and the efficiency of the methods as discussed in Section 3.2.

As observed from the results, the total number of the iterations required in the SBO method is very low.

The accuracy and the computational efficiency of the SBO method with ECB+CA Automated approach is very satisfactory for the selected problem.

\section{Summary and conclusions}

The contribution of this research is proposing solutions to one of the major difficulties, analysis time, in structural optimization by taking the advantage of effective structural reanalysis techniques in an SBO scheme.

Integration of two reanalysis techniques into the CraigBampton (CB) method is introduced. The Enriched CB (ECB) method and the Combined Approximations (CA) approach are used for approximate computation of the fixed interface and the constraint modes of the CB transformation matrix, respectively. The ECB method extends the fixed interface normal mode set by including the effects of the residual forces acting on the modified structure. Thus modal analysis can be avoided while preserving the accuracy to a certain extent. Unfortunately, there is no proposed automated update scheme in the literature that switches the ECB method with the exact analysis when the accuracy and the computational accuracy are not balanced. The automated computation of the constraint modes is a very attractive feature in which the exact or the approximate calculation of the modes is automatically decided using the number of FLOPs. These are then used at the analysis step of a Surrogate-Based Optimization strategy for improving the computational efficiency during optimization. The strategy 
is demonstrated by an academic test problem. The selected structure has repeating patterns in its geometry. The reduced FE model of the complete structure is generated by the parameterized model of one component. The reduced matrices of each computed component configuration are kept in the libraries. When similar components need to be analyzed again, the corresponding reduced matrices are called from the libraries. Hence, unnecessary static and dynamic analyses required for the calculation of the $\mathrm{CB}$ transformation matrix are prevented.

The results of the test case are very promising for the application of the proposed strategy on small-scale sizing optimization problems where the dynamic behavior of large and/or complex structures is required to be modified. The computational efficiency of the SBO method improves when the proposed reanalysis methods are used for the calculation of the $\mathrm{CB}$ transformation matrix at the analysis step. Moreover, the accuracy of the provided solutions is preserved. It is believed that the efficiency of the strategy will be more pronounced when tested on more complex problems.

Open Access This article is distributed under the terms of the Creative Commons Attribution Noncommercial License which permits any noncommercial use, distribution, and reproduction in any medium, provided the original author(s) and source are credited.

\section{References}

Akçay Perdahcıŏ̆lu D, Ellenbroek MHM, van der Hoogt PJM, de Boer A (2009) An optimization method for dynamics of structures with repetitive component patterns. Struct Multidisc Optim 39(6):557567

Akçay Perdahcıŏglu D, Ellenbroek MHM, Geijselaers HJM, de Boer A (2010) Updating the Craig-Bampton reduction basis for efficient structural reanalysis. Int J Numer Methods Eng doi:10.1002/nme. 2983

Barthelemy JFM, Haftka RT (1993) Optimization of large structural systems. Kluwer, Dordrecht

Benfield WA, Hruda RF (1971) Vibration analysis of structures by component mode substitution. AIAA 9(7):1255-1261

Berke L, Hajela P (1992) Application of neural nets in structural mechanics. Struct Optim 4:90-98

Booker AJ, Dennis JE Jr, Frank PD, Serafini DB, Torczon V, Trosset MW (1998) A rigorous framework for optimization of expensive functions by surrogates. ICASE Report. NASA, Langley Research Center, pp 98-47
Bouazzouni A (1997) Selecting a ritz basis for the reanalysis of the frequency response functions of modified structures. J Sound Vib 198(2):309-322

Cerulli C, van Keulen F, Rixen DJ (2007) Dynamic reanalysis and component mode synthesis to improve aircraft modeling for load calculation. In: 48th AIAA/ASME/ASCE/AHS/ASC structures, structural dynamics, and materials conference

Craig RR, Bampton MCC (1968) Coupling of substructures for dynamic analysis. AIAA 6(7):1313-1319

Goldman RL (1969) Vibration analysis by dynamic partitioning. AIAA $7: 1152-1154$

Haftka RT, Gürdal Z (1992) Elements of Structural Optimization, vol 11, 3rd edn. Kluwer Academics Publishers

Hurty WC (1965) Dynamic analysis of structural systems using component modes. AIAA 3(4):678-685

Jacobs J, Etman L, van Keulen F, Rooda J (2004) Framework for sequential approximate optimization. Struct Multidisc Optim 27

Jones D, Schonlau M, Welch W (1998) Efficient global optimization of expensive black-box functions. Global Optimization 13(4):455492

Kirsch U (1994) Efficient sensitivity analysis for structural optimization. Comput Methods Appl Mech Eng 117:143-156

Kirsch U (2002) Design Oriented Analysis of Structures. A Unified Approach, Solid Mechanics and its Applications, vol 95. Springer

Kirsch U (2008) Reanalysis of Structures. A Unified Approach for Linear, Nonlinear, Static and Dynamic Systems, Solid Mechanics and its Applications, vol 151. Springer

Kirsch U, Bogomolni M (2004) Procedures for approximate eigenproblem reanalysis of structures. Int J Numer Methods Eng 60:1969-1986

Mac Neal RH (1971) A hybrid method of component mode synthesis. Comput Struct 1(4):581-601

Mackay D (1992) A practical bayesian framework for backpropagation networks. Neural Comput 4:448-472

Markovic D, Park K, Ibrahimbegovic A (2007) Reduction of substructural interface degrees of freedom in flexibility based component mode synthesis. Int J Numer Methods Eng 70:163-180

Masson G, Ait Brik B, Cogan S, Bouhaddi N (2006) Component mode synthesis (cms) based on an enriched ritz approach for efficient structural optimization. J. Sound Vib. 296:845-860

Maute K, Nikbay M, Farhat C (2001) Coupled analytical sensitivity analysis and optimization of three-dimensional nonlinear aeroelastic systems. AIAA 39(11):2051-2061

Queipo N, Haftka R, Shyy W, Goel T, Vaidyanatan R, Tucker P (2005) Surrogate-based analysis and optimization. Aerosp Sci 41:1-28

Rixen DJ (2002) A dual craig-bampton method for dynamic substructuring. J Comput Appl Math 168(1-2):383-391

Rubin S (1975) Improved component mode representation for structural dynamic analysis. AIAA 13(8):995-1006

Svanberg K (1987) The method of moving asymptotes-a new method for structural optimization. Int J Numer Methods Eng 24:359373

Van Keulen F, Haftka R, Kim N (2005) Review of options for structural design sensitivity analysis. part 1: Linear systems. Comput Methods Appl Mech Eng 194:3213-3243 